IOS Press

\title{
Editorial
}

\section{Special issue: Mobile web data analytics (part I)}

\author{
Zili Zhang ${ }^{\text {a,* }}$, Li Liu ${ }^{\mathrm{b}}, \mathrm{Li} \mathrm{Li}^{\mathrm{a}}{ }^{\mathrm{a},}$, and Xiangliang Zhang ${ }^{\mathrm{c}}$ \\ a Southwest University, China \\ E-mails: zili.zhang@deakin.edu.au,lily@swu.edu.cn \\ ${ }^{\mathrm{b}}$ Chongqing University, China \\ ${ }^{c}$ King Abdullah University of Science and Technology, Saudi Arabia
}

It is essential to constantly collect data with various mobile applications from diverse sources, such as smartphones and ubiquitous sensors. However, how do you conduct the analysis on such a mass of mobile data or mobile web data aiming to solve issues in different areas of applications, including human behavior recognition, medication, recommendation and transportation? Nowadays, research in mobile and social computing environments is now turning to novel concepts to address the challenge of data processing and analyzing. The special issue Mobile web data analytics addresses issues of data management in mobile and social computing environments with a special focus on data processing and applications. The goal of the special issue is to build a forum for researchers from academy and industry to investigate challenging and innovative research issues on the subject, which combines data analytics within mobile and social environment and to explore creative concepts, theories, innovative technologies and intelligent solutions. We intend this special issue to act as an initial place where people from different areas can find a forum to discuss issues of data management and processing in new and emerging mobile computing environments.

We accepted 11 papers that provide deep research results to report the advance of mobile web data analytics and applications. These papers are grouped into

\footnotetext{
*Corresponding authors. E-mails: zili.zhang@deakin.edu.au, lily@swu.edu.cn.
}

two special issues. This first special issue contains 5 papers.

In this special issue, the first contribution is " $H o t$ time periods discovery for facility proportioning in urban commercial districts using POIs and mobile phone data" by Xie et al., which presented an approach to mining hot time periods in urban commercial districts using POI information and mobile phone data, which is valuable for the analysis of activity regularity of population in urban cities. The second paper "A point of interest recommendation method using user similarity" by Zeng et al. proposed an efficient POI recommendation method that finds out users' similarities using diverse data including their locations data to form an ordered POI recommendation list. The third paper "Content tracking by leveraging hashtag and time information in Twitter social media" by Xu et al. developed two methods to detect and track the evolution of content in Twitter social media by integrating hashtag and time information. The advantage of the proposed methods is that it captures the hashtags distribution over topics and topic changes over time simultaneously. In the fourth paper "Finger gesture recognition using a smartwatch with integrated motion sensors", Li et al. utilized data collected from the sensors of accelerometer and gyroscope embedded in smartwatch to recognize finger gestures. Their results show the viability of recognizing finger or hand gestures using motion sensors. The fifth paper " $u$ Gait: a platform for automated quantitative gait analysis and its application to Parkinson's disease" by $\mathrm{Wu}$ et al. presented their 
gait sensing platform that can discriminate Parkinson patients from healthy subjects by recognizing the patterns of their movements using motion sensors. Their experimental results show the capability of recognizing a variety of gait parameters improving the classification of different movements of Parkinson patients and healthy persons.

In summary, the goal of this special issue is to crystallize the emerging mobile web data technologies and trends into positive efforts to focus on the most promising solutions in applications. The papers show that mobile data technologies play a more and more important role in various applications. This special issue intends to act as an initial place for people from different areas to discuss issues in emerging mobile computing environments.

\section{Biographies}

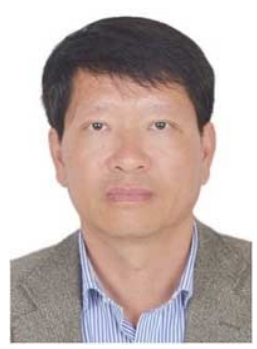

Zili Zhang is a professor at Southwest University, Chongqing, China, and a senior lecturer at the School of Information Technology at Deakin University. He received his BSc from Sichuan University, MEng from Harbin Institute of Technology, and Ph.D. from Deakin University, all in computing. He authored or co-authored over 160 refereed papers in international journals and conference proceedings, one monograph, and seven textbooks. His research interests include bio-inspired artificial intelligence, agent-based computing, and big data analysis. $\mathrm{He}$ is a distinguished member of Chinese Computer Federation (CCF).

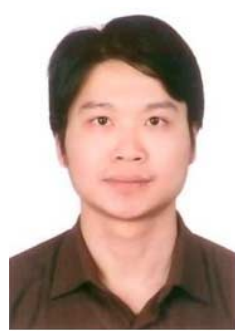

Li Liu is an associate professor at Chongqing University. He also serves as a Senior Research Fellow of the School of Computing at the National University of Singapore. $\mathrm{Li}$ received his Ph.D. in Computer Science from the Université Parissud XI in 2008. He has served as an associate professor at Lanzhou
University in China. His research interests include pattern recognition, data analysis, and their applications on human behaviors. He aims to contribute in interdisciplinary research of computer science and human related disciplines. Li has published widely in conferences and journals with over 50 peer-reviewed publications. Li has been the Principal Investigator of several funded projects from government and industry.

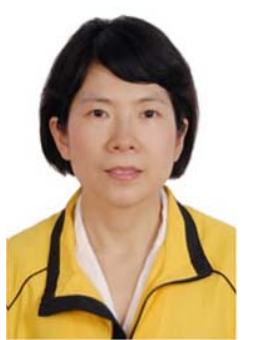

$\mathbf{L i ~} \mathbf{L i}$ is a professor at the School of Computer and Information Science at Southwest University, Chongqing, China. She received her Ph.D. degree in Computer Science from Swinburne University of Technology, Melbourne, Australia in 2006. Her research interests include data analysis, data mining and machine learning. She has published over 70 peerreviewed papers. She is a senior member of China Computer Federation and Chinese Association for Artificial Intelligence.

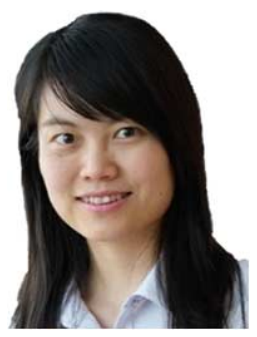

Xiangliang Zhang is an associate professor at King Abdullah University of Science and Technology. She earned her Ph.D. degree in computer science from the INRIA-Universite Paris-Sud, France, in July 2010. She received M.S. and B.S. degrees from Xi' an Jiaotong University, China, in 2006 and 2003, respectively. Her research mainly focuses on developing automated methods for machine learning and data mining to discover and manage knowledge from complex and large-scale data sets for diverse applications. She has authored or coauthored over 40 research papers in referred international journals and conference proceedings. She is the guest editor of Mathematics in Computer Sciences and IEEE Network. She serves as Technical Program Committee member for SIGKDD, ICDE, ICDM, CIKM, etc. 